\section{Healthy lifestyle and life expectancy free of chronic diseases}

The average life expectancy in the world has increased substantially in the past few decades.Modifiable lifestyle factors including smoking, physical activity, alcohol intake, body weight, and diet quality affect both total life expectancy and incidence of chronic diseases. Few studies have comprehensively examined how a combination of multiplelifestyle factors may relate to life expectancy free from major diseases suchas diabetes, cardiovascular disease, and cancer. A Harvard teamexamined the effect of healthy lifestyle factors on life expectancy free of chronic diseases, using data from up to 34 years of follow-up in the Nurses' Health Study (NHS) (1980-2014; n=73 196) and 28 years of follow-up in the Health Professions Follow-up Study (HPFS)(1986-2014;n=38 366).A healthy lifestyle score based oninformation on five lifestyle factorsdiet, smoking,physical activity, alcohol consumption, and body massindex (BMI) was derived.Five low-risk lifestyle factors included: never smoking, bodymass index 18.524.9 , moderate to vigorous physicalactivity $(\geq 30$ minutes/day), moderate alcohol intake(women: 5-15 g/day; men 5-30 g/day), and a higherdiet quality score (upper $40 \%$ ). Women who met all the healthful lifestyle measures had an additional 10.7 years of disease-free life compared with women who met no healthful lifestyle measures. For men, the number was 7.6 additional disease-free years. So it was concluded that a healthier lifestyle was associated with an increased total life expectancy and life expectancy free of cancer, cardiovascular disease, and type 2 diabetes. Findings suggest thatthe promotion of a healthy lifestyle would help to reduce healthcare burdens. Public policiesfor improving food and the physical environmentconducive to adopting a healthy diet and lifestyleare critical to improving life expectancy, especially lifeexpectancy free of major chronic diseases.

Source:BMJ 2020; 368:16669 http://dx.doi.org/10.1136/bmj.16669

\section{Prenatal and Childhood Antibiotic Exposure and Obesity at Age 4 Years}

Although antibiotics are associated with obesity in animal models, the evidence in humans is conflicting. To assess whether antibiotic exposure during pregnancy and/or early childhood is associated with thedevelopment of childhood, a cross-sectional studywas conducted in New Zealand. The overall study population consisted of 132852 mothers and 151359 children.Results from covariate-adjusted analyses showed that both prenataland early childhood exposures to antibiotics were independently associated with obesity at age 4 years, in adose-dependent manner. Every additional course of antibiotics dispensed to the mothers yielded an adjustedodds ratio (aOR) of obesity in their children of 1.02 . For the child'sexposure, the aOR for the association between antibiotic exposure and obesity was 1.04 among siblings and 1.05 among twins. However, fixed-effects analyses ofsiblings and twins showed no associations between antibiotic exposure and obesity, with aORs of 0.95for maternal exposure, 1.02 for child's exposure, and 0.91 for twins' exposure. So although initial analyses on the whole population, siblings, and twins demonstrated an association betweenantibiotic exposure and odds of obesity, further detailed analyses of siblings and twins with discordantoutcomes showed no associations. These discordant results likely reflect unmeasured confounding factors. Therefore, although judicious use of antibiotics is necessary, antibiotics are unlikely to be a majorcontributor to childhood obesity.

Source: JAMA Netw Open. 2020 Jan; 3(1): e1919681.

\section{Landmark study to transform cancer treatment}

More than a thousand scientists have built the most detailed picture of cancer ever in a landmark study. They said the cancer was like a 100,000-piece jigsaw, and that until today, $99 \%$ of the pieces were missing. Their studies provide an almost complete picture of all cancers. They could allow treatment to be tailored to each patient's unique tumor, or develop ways of finding cancer earlier. The Pan-Cancer Analysis of Whole Genomes Consortium analyzed the whole genetic code of 2,658 cancers. The work, which took 22 scientific journal papers to describe, shows that cancer is massively complex, with thousands of different combinations of mutations able to cause cancer. The project found people's cancers contain, on average, between four 
and five fundamental mutations that drive cancer's growth. These are potential weak-spots that can be exploited with treatments that attack these "driver mutations". However, $5 \%$ of cancers appear to have no driver mutations at all, showing there is still more work to do. Scientists also developed a way of "carbon dating" mutations. They showed that more than a fifth of them occurred years or even decades before cancer is found. They have developed the first timelines of genetic mutations across the spectrum of cancer types. Furthermore, unlocking these patterns means it should now be possible to develop new diagnostic tests that pick up signs of cancer much earlier. Now the challenge will be knowing which of these mutations will go on to become cancer and which can be safely ignored.

Source:Nature 2020:578. https://doi.org/10.1038/s41586020-1969-6

\section{Enzalutamide with Standard Therapy in MetastaticProstate Cancer}

Enzalutamide, an androgen-receptor inhibitor, has been associated with improved overall survival in men with castration-resistant prostate cancer. In an open-label, randomized, phase 3 trial, patients were assigned to receive testosterone suppression plus either open-label enzalutamide or a standard nonsteroidal antiandrogen therapy (standard-care group). A total of 1125 men underwent randomization; the median follow-up was 34 months. The primary endpoint was overall survival. Secondary endpoints included progression-free survival as determined by the prostate-specific antigen (PSA) level, clinical progression-free survival, and adverse events. There were 102 deaths in the enzalutamide group and 143 deaths in the standard-care group $(\mathrm{P}=0.002)$. KaplanMeier estimates of overall survival at 3 years were $80 \%$ in the enzalutamide group and $72 \%$ in the standardcare group. Better results with enzalutamide were also seen in PSA progression-free survival $(\mathrm{P}<0.001)$ and in clinical progression-free survival $(\mathrm{P}<0.001)$. However, treatment discontinuation due to adverse events was more frequent in the enzalutamide group than in the standardcare group (33 events and 14 events, respectively). Fatigue was more common in the enzalutamide group; seizures occurred in 7 patients in the enzalutamide group (1\%) and in no patients in the standardcare group. Hence, it was concluded that enzalutamide was associated with significantly longer progression-free and overall survival than standard care in men with metastatic, hormone-sensitive prostate cancer receiving testosterone suppression.

Source: N Engl J Med 2019; 381:121-131. DOI: 10.1056/NEJMoa1903835.

\section{Clinical Characteristics of Coronavirus Disease 2019 in China}

In early December 2019, the first pneumonia cases of unknown origin were identified in Wuhan, the capital city of the Hubei province of China. The pathogen has been identified as a novel enveloped RNA betacoronavirus that has currently been named severe acute respiratory syndrome coronavirus 2 (SARS-CoV-2). The World Health Organization (WHO) has recently declared coronavirus disease 2019 (Covid-19) a public health emergency of international concern. By the end of February 2020, a total of 81,109 laboratory-confirmed cases had been documented globally.Since December 2019, when coronavirus disease 2019 (Covid-19) emerged in Wuhan city and rapidly spread throughout China, data have been needed on the clinical characteristics of the affected patients. Data wasextracted regarding 1099 patients with laboratory-confirmed Covid19 from 552 hospitals in 30 provinces, autonomous regions, and municipalities in mainland China through January 29, 2020. The primary composite endpoint was admission to an intensive care unit (ICU), the use of mechanical ventilation, or death. The median age of the patients was 47 years $(41.9 \%$ were females). The primary composite endpoint occurred in 67 patients $(6.1 \%)$, including $5.0 \%$ who were admitted to the ICU, $2.3 \%$ who underwent invasive mechanical ventilation, and $1.4 \%$ who died. Only $1.9 \%$ of the patients had a history of direct contact with wildlife. Among nonresidents of Wuhan, $72.3 \%$ had contact with residents of Wuhan, including $31.3 \%$ who had visited the city. The most common symptoms were fever $(43.8 \%$ on admission and $88.7 \%$ during hospitalization) and cough (67.8\%). Diarrhea was uncommon (3.8\%). The median incubation period was 4 days (range 2 to 7 ). On admission, ground-glass opacity was the most common radiologic finding on chest computed tomography (CT) (56.4\%). No radiographic or CT abnormality was found in 157 of 877 patients (17.9\%) with nonsevere disease and in 5 of 173 patients (2.9\%) with 
severe disease. Lymphocytopenia was present in $83.2 \%$ of the patients on admission.

Source:DOI: 10.1056/NEJMoa200203

Is pre-operative dysglycemia associated with decreased survival in patients

with pancreatic neuroendocrine neoplasms?

Diabetes mellitus is associated with an increased risk of pancreatic cancer and impaired post-resection survival. However, such evidence is lacking for pancreatic neuroendocrine neoplasms in diabetes mellitus patients. With the aim to evaluate the glycemic profile and to assess the potential impact of glycemic control on long-term outcomes in patients undergoing resection of pancreatic neuroendocrine neoplasms, pancreatic resections from 2001 to 2017 for pancreatic neuroendocrine neoplasms were analyzed from prospective databases. Blood glucose and $\mathrm{HbA} 1 \mathrm{c}$ levels were collected from preoperative tests. Preoperative dysglycemia was defined as a blood glucose $\geq 140 \mathrm{mg} \%$ and/or HbA1c $\geq 6.5 \%$. Four hundred and seventeen patients were analyzed. No differences regarding outcomes were detected between diabetics and nondiabetics. Conversely, patients with dysglycemia had greater rates of metastasis $(16.8 \%$ vs $27.4 \% ; \mathrm{P}=.027)$ as well as vascular, perineural, and lymphovascular involvement than those with normal blood glucose $(89.2 \%$ vs $57.4 \% ; \mathrm{P}<.001,90.0 \%$ vs $65.1 \% ; \mathrm{P}=.046$, and $89.3 \%$ vs $61.3 ; \mathrm{P}=.006$, respectively). Preoperative dysglycemia was associated with impaired overall survival and recurrencefree survival. By multivariate analysis, preoperative dysglycemia was independently associated with recurrence-free survival, together with lymph-node involvement and metastatic disease. In conclusion,preoperative dysglycemia, but not diabetes mellitus per se, is associated with advanced disease and impaired long-term outcomes in patients undergoing resection for a pancreatic neuroendocrine neoplasm. For those patients, closer surveillance and strict glycemic control are warranted.

Source: DOI: https://doi.org/10.1016/j.surg.2019.11.007

\section{Peerzada Umar Farooq Baba} Adil Hafeez Wani 